\title{
EDITORIAL
}

For reprint orders, please contact: reprints@futuremedicine.com

\section{Fibrosis, cancer and the premetastatic niche}
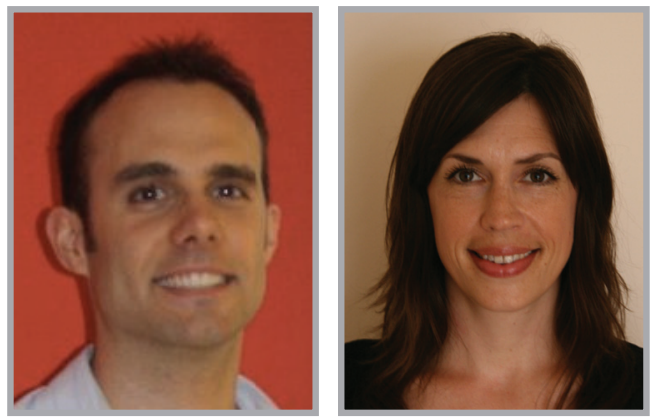

"Knowing who, when and how long to treat with antifibrotic therapies in the cancer context may prove problematic as the long-term side effects of such treatments are currently unknown.”

Thomas R Cox ${ }^{*, 1} \&$ Janine T Erler ${ }^{*, 1}$

The onset and development of breast cancer is known to be a complex process involving reciprocal interactions between multiple cell types within the body. Epithelial, endothelial, mesenchymal and hematopoietic cells act together to alter and shape the microenvironment of the tumor to modulate the initiation, progression and dissemination of cancer. Their actions result in a dramatically altered extracellular matrix (ECM) from both a biochemical, but also biomechanical context. As such, over the past decade, solid tumors have become increasingly recognized as discrete organs, showing a complexity that approaches and perhaps even exceeds that of healthy tissue. These significant changes are the result of normal homeostatic mechanisms gone awry and as such make targeting them incredibly difficult.

Tumors develop as a result of the dysregulation of multiple signaling networks governing fundamental cell processes such as apoptosis, proliferation, differentiation and migration. All of these cellular processes are inherently important to tumor initiation, progression and metastasis. One of the critical regulators of these is the ECM, and more importantly, ECM dynamics within the tumor microenvironment. Biological regulation of cellular behavior and phenotype by the ECM is rapidly emerging as a major research area. The ability of cells to sense and respond to changes in biochemical and biomechanical cues provided by ECM is now considered an important factor in determining cancer onset and progression to metastasis.

Under normal conditions, homeostasis, the secretion of new and breakdown of old ECM components is tightly controlled, and this balance helps to maintain normal order and structure within organs. Early on in tumorigenesis, this homeostasis is upset and the ECM begins to change in both its biochemical as well as its biomechanical properties. In the case of breast cancer, it has been shown that these desmoplastic changes, driven primarily by collagen I accumulation, are a key component of the tumor microenvironment [1] and act to drive malignant transformation [2], but also drive tumor progression $[3,4]$ and ultimately metastasis $[5,6]$. Yet this disruption to homeostatic ECM mechanisms is not confined to the primary tumor site. Indeed, the tumor is capable of eliciting changes within the ECM in sites of future

'Biotech Research \& Innovation Centre (BRIC), University of Copenhagen, Copenhagen, Denmark

*Authors for correspondence: thomas.cox@bric.ku.dk; janine.erler@bric.ku.dk

\section{KEYWORDS \\ - fibrosis • LOX • metastasis \\ - microenvironment $\bullet$ niche}

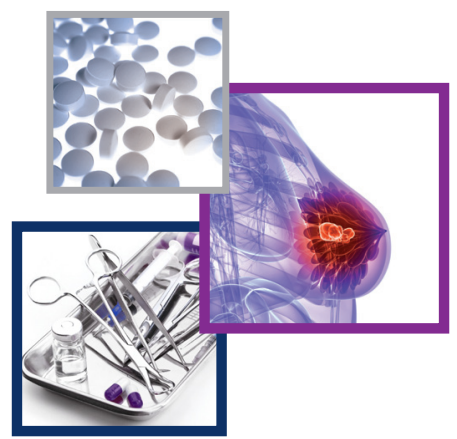

\footnotetext{
"The ability of cells to sense and respond to changes in biochemical and biomechanical cues provided

by extracellular matrix is now considered an important factor in determining cancer onset and progression to metastasis."
} 
"... it is likely that we will be able to block the pro-tumorigenic and pro-metastatic changes in the extracellular matrix without disrupting normal regenerative processes to improve the prognosis and outcome of breast cancer patients." metastasis, so-called premetastatic niches [7-10]. Many of these changes we see during the formation of premetastatic niches; the recruitment of immune cells, the secretion of ECM components and ECM-modifying enzymes, are highly reminiscent of changes observed in another pathological disease, namely that of fibrosis.

Fibrosis is a huge clinical problem, and affects millions of people worldwide. The links between cancer and fibrosis are strong. Indeed, it is well known that underlying tissue fibrosis is closely correlated with the onset of cancer in organs such as the liver and the lung. For example, it has been shown that fibrotic breast disease is associated with a predisposition to breast cancer [11], and environmentally induced fibrotic disorders of the lung can increase incidence of lung cancer [12]. To that end, it has been proposed that the treatment of underlying fibrosis in such cases would lead to an improvement in cancer associated prognosis [13]. In our work last year, we showed that tissue fibrosis may pose a serious clinical problem in patients harboring unrelated primary tumors [6]. The presence of pathological organ fibrosis in both the lung and the liver increases the ability of circulating breast cancer cells to colonize and grow in these organs, suggesting that breast cancer patients with unrelated organ fibrosis may present a poorer metastasis-free survival. This work is supported by others who showed that in the liver, myofibroblast activation is associated with the arrival of melanoma cells into the liver, leading to enhanced colonization of the organ [14]. That the onset of tissue fibrosis is capable of modulating tumor cell behavior at secondary sites is also supported by work showing that developing tissue fibrosis is capable of re-activating already disseminated dormant tumor cells [15]. Together, this body of work illustrates how collagen remodeling and changes in both the biochemical as well as biomechanical properties of the local microenvironment may be capable of modulating the behavior of tumor cells at secondary metastatic sites.

Given the significant overlap in pathways associated with fibrosis and cancer, we should simultaneously evaluate agents that target the tumor microenvironment and fibrosis for potential roles in both settings. When considering adjuvant therapies for metastatic breast cancer, just as antifibrotic therapies are being proposed for highly fibrotic primary tumors, it may also be necessary to treat patients with antifibrotic therapies aimed at disrupting the establishment and development of metastases. Even better would be to target commonalities in mechanisms behind tumor initiation and progression and the onset and development of fibrosis. A key family shown to play a pivotal role in these two diseases in the lysyl oxidase (LOX) family of proteins, in particular LOX and LOX-like 2 (LOXL-2) [16,17]. Both of these proteins have been shown to be important in ECM remodeling and homeostasis, and to play critical roles in both breast cancer and fibrosis [6,7,18-20] driving the accumulation of collagen fibers. Indeed, a role for LOX and LOXL-2 has been shown in chronic hepatic fibrosis, arterial fibrosis and chemotherapy-induced kidney fibrosis in human patients [21], as well as liver and lung fibrosis in mouse models [6,18]. While targeting fibrosis has proved problematic in the past, several therapeutic approaches are being explored and clinical trials with a LOXL-2 targeting antibody [18] are in Phase II for fibrosis and beginning in cancer patients. Data would suggest that as well as targeting the primary tumor cells directly, these therapies would also disrupt aberrant remodeling of the ECM at the primary tumor site and secondary metastatic sites thereby improving the prognosis of patients. Recent work by Gilkes and colleagues has also shown that primary tumor hypoxia leads to the increased breast cancer tumor cell secretion of collagen prolyl hydroxylases that are critical for collagen deposition and desmoplasia, and leads to enhanced metastasis to lymph nodes and lungs in human xenograft models [22].

While we are still uncovering more about the interplay between organ fibrosis and cancer metastasis, it is clear that new challenges are yet to emerge. For example, blocking aberrant changes in the ECM may prove beneficial, but sustained inhibition may adversely affect normal programs of wound healing. Knowing who, when and how long to treat with antifibrotic therapies in the cancer context may prove problematic as the long-term side effects of such treatments are currently unknown. However, in time it is likely that we will be able to block the pro-tumorigenic and pro-metastatic changes in the ECM without disrupting normal regenerative processes to improve the prognosis and outcome of breast cancer patients.

\section{Author contributions}

Written by TR Cox, revised by JT Erler; final version approved by TR Cox and JT Erler. 


\section{Financial \& competing interests disclosure}

The authors would like to thank BRIC, the Novo Nordisk Foundation (Hallas Møller Stipendium to JT Erler), the Danish Cancer Society, and the Danish Ministry for Science, Innovation and Higher Education for financial support to the Erler laboratory. The authors have no other relevant affiliations or financial involvement with any organization or entity with a financial interest in or financial conflict with the subject matter or materials discussed in the manuscript apart from those disclosed.

No writing assistance was utilized in the production of this manuscript.

\section{References}

1 Egeblad M, Rasch MG, Weaver VM. Dynamic interplay between the collagen scaffold and tumor evolution. Curr. Opin. Cell Biol. 22(5), 697-706 (2010).

2 Levental KR, Yu H, Kass L et al. Matrix crosslinking forces tumor progression by enhancing integrin signaling. Cell 139(5), 891-906 (2009).

3 Baker AM, Bird D, Welti JC et al. Lysyl oxidase plays a critical role in endothelial cell stimulation to drive tumor angiogenesis. Cancer Res. 73(2), 583-594 (2013).

4 Baker AM, Cox TR, Bird D et al. The role of lysyl oxidase in SRC-dependent proliferation and metastasis of colorectal cancer. J. Natl Cancer Inst. 103(5), 407-424 (2011).

5 Baker AM, Bird D, Lang G, Cox TR, Erler JT. Lysyl oxidase enzymatic function increases stiffness to drive colorectal cancer progression through FAK. Oncogene 32(14), 1863-1888 (2012).

6 Cox TR, Bird D, Baker AM. LOX-mediated collagen crosslinking is responsible for fibrosis-enhanced metastasis. Cancer Res. 73(6), 1721-1732 (2013).

7 Erler JT, Bennewith KL, Cox TR. Hypoxiainduced lysyl oxidase is a critical mediator of bone marrow cell recruitment to form the premetastatic niche. Cancer Cell 15(1), 35-44 (2009).

8 Kaplan RN, Riba RD, Zacharoulis S. VEGFR1-positive haematopoietic bone marrow progenitors initiate the pre-metastatic niche. Nature 438(7069), 820-827 (2005).

9 Hiratsuka S, Watanabe A, Aburatani H, Maru Y. Tumour-mediated upregulation of chemoattractants and recruitment of myeloid cells predetermines lung metastasis. Nat. Cell Biol. 8(12), 1369-1375 (2006).

10 Hiratsuka S, Ishibashi S, Tomita T. Primary tumours modulate innate immune signalling to create pre-metastatic vascular hyperpermeability foci. Nat. Commun. 4, 1853 (2013).

11 Jacobs TW, Byrne C, Colditz G, Connolly JL, Schnitt SJ. Radial scars in benign breastbiopsy specimens and the risk of breast cancer. N. Engl. J. Med. 340(6), 430-436 (1999).

12 Mossman BT, Churg A. Mechanisms in the pathogenesis of asbestosis and silicosis. Am. J. Respir. Crit. Care Med. 157(5 Pt 1), 1666-1680 (1998).

13 Bilimoria MM, Lauwers GY, Doherty DA et al.; International Cooperative Study Group on Hepatocellular Carcinoma. Underlying liver disease, not tumor factors, predicts long-term survival after resection of hepatocellular carcinoma. Arch. Surg. 136(5), 528-535 (2001).

14 Olaso E, Santisteban A, Bidaurrazaga J, Gressner AM, Rosenbaum J, VidalVanaclocha F. Tumor-dependent activation of rodent hepatic stellate cells during experimental melanoma metastasis. Hepatology 26(3), 634-642 (1997).
15 Barkan D, El Touny LH, Michalowski AM et al. Metastatic growth from dormant cells induced by a col-I-enriched fibrotic environment. Cancer Res. 70 (14), 5706-5716 (2010).

16 Cox TR, Erler JT. Remodeling and homeostasis of the extracellular matrix: implications for fibrotic diseases and cancer. Dis. Model Mech. 4(2), 165-178 (2011).

17 Barker HE, Cox TR, Erler JT. The rationale for targeting the LOX family in cancer. Nat. Rev. Cancer 19(12), 540-552 (2012).

18 Barry-Hamilton V, Spangler R, Marshall D et al. Allosteric inhibition of lysyl oxidaselike-2 impedes the development of a pathologic microenvironment. Nat. Med. 16(9), 1009-1017 (2010).

19 Barker HE, Chang J, Cox TR et al. LOXL2-mediated matrix remodeling in metastasis and mammary gland involution. Cancer Res. 71(5), 1561-1572 (2011).

20 Erler JT, Bennewith KL, Nicolau M et al. Lysyl oxidase is essential for hypoxia-induced metastasis. Nature 440 (7088), 1222-1226 (2006).

21 Maki JM. Lysyl oxidases in mammalian development and certain pathological conditions. Histol. Histopathol. 24(5), 651-660 (2009).

22 Gilkes DM, Chaturvedi P, Bajpai S. Collagen prolyl hydroxylases are essential for breast cancer metastasis. Cancer Res. 73(11), 3285-3296 (2013). 